\title{
Combining enhanced recovery and short-stay protocols for hip and knee joint replacements: the ideal solution
}

\author{
Pascal-André Vendittoli, MD, \\ MSc \\ Karina Pellei, PTH, MSc \\ Carla Williams, MHSM, CPPS \\ Claude Laflamme, MD, MHSc
}

Accepted March 17, 2020

\section{Correspondence to:}

P.-A. Vendittoli

Hôpital Maisonneuve-Rosemont

5415 Boul L'Assomption

Montréal QC H1T 2M4

pa.vendittoli@me.com

DOI: $10.1503 /$ cjs.019519

\section{SUMMARY}

Pressure to reduce health care costs, limited hospital bed availability as well as improvements in surgical techniques and perioperative care motivated many health care centres to implement short-stay protocols for patients undergoing hip or knee arthroplasty. To improve patient outcomes and maintain care safety, we strongly believe the best way to implement a successful outpatient program would be to embrace the principles of Enhanced Recovery After Surgery (ERAS), and to improve patient recovery to a level such that the patient could leave the hospital sooner. Enhanced Recovery Canada and the Canadian Patient Safety Institute support the development of ERAS pathways for orthopedic procedures. The goal is to provide patients, health care providers and leaders with helpful tools and resources to effectively implement and sustain ERAS protocols. Reducing the rate of adverse events while reducing the length of hospital stays to less than 24 hours is a winning situation for everyone.

$\mathrm{n}$ recent years, there has been a shift toward outpatient and short-stay protocols for patients undergoing total hip arthroplasty (THA) or total knee arthroplasty (TKA). This is due, in part, to improvements in surgical technique and perioperative care, but also in response to pressure to reduce health care costs. ${ }^{1}$ Discharging patients home earlier may be possible either by transferring part of in-hospital care to the patient home or by implementing different strategies to avoid program failures (e.g., the use of peripheral nerve block with continuous local anesthetics infiltration, large wound dressings to prevent dressing change, prescription of long-acting opioids, prolonged use of walking aids). Using such strategies, some short-stay protocols have shifted away from aiming to reduce complications and improved recuperation to instead using length-of-stay as the main measure of success. However, success for patients is determined by improved well-being, improved function and reduced complication rates. The most appropriate way to implement a successful outpatient surgical program would be to apply the principles of Enhanced Recovery After Surgery (ERAS), and to improve patient recovery to a level where patients could leave the hospital sooner. ${ }^{2}$

\section{WHAT IS ERAS?}

Dr. Husted and Dr. Kehlet pioneered the field of perioperative care and developed the ERAS program. ${ }^{2}$ The program aims to reduce the duration of hospital stay for patients undergoing major surgery by providing optimized care. However, achieving outpatient surgery is a secondary objective of ERAS. The ERAS Society has issued a consensus statement for perioperative care for patients undergoing THA and TKA. ${ }^{3}$ Main aspects of the ERAS protocol include patient education and optimization, pain control, nutrition and gastrointestinal function, mobilization, blood conservation and reduction of adverse events. 


\section{Preoperative patient optimization}

Preoperative education is an essential part of a patient's preparation for surgery. It is designed to improve the patient's knowledge, expectations, behaviour and health. Helpful patient engagement animations can be found at https://precare.ca/erc. The preoperative period also provides an opportunity for patients to optimize modifiable risk factors, including nutrition and cessation of smoking and alcohol, and for surgeons to coach patients in pre- and postoperative exercises.

\section{Pain control}

Postoperative pain is unavoidable and, depending on severity, can affect functional recovery, reduce patient satisfaction and prolong the duration of hospital stay. Postoperative pain control has traditionally been managed by opioids, known to produce adverse effects like drowsiness, nausea, vomiting, constipation and respiratory depression. A multimodal analgesia approach should be implemented to minimize or avoid opioid use. ${ }^{3-5}$ The ERAS multimodal approach to pain control includes patient engagement and education, a pre-emptive medication cocktail, an epidural-sedation anesthesia combination, wound infiltration of local anesthetics, tourniquet avoidance for TKA and postoperative cryotherapy.

\section{Gastrointestinal function}

Postoperative nausea and vomiting delay eating and mobilization, and impede recovery. There are many causes of postoperative nausea, including the combination of various anesthetic agents, hypovolemia, prolonged perioperative fasting, anemia, immobilization and opioid use. The ERAS multimodal approach includes an improved presurgery feeding protocol, the use of prophylactic drugs to reduce nausea and vomiting (e.g., dexamethasone, aprepitant or a scopolamine patch), as well as opioid avoidance and early mobilization. ${ }^{3,5}$ Moreover, patients are encouraged to eat as soon as desired after surgery.

\section{Early mobilization}

Early postoperative function and mobilization are key factors of a short-stay program. They can be facilitated by a combination of preoperative education, pain and nausea control and avoiding the use of catheterization and drains. Moreover, anesthesia techniques should minimize perioperative motor block, autonomic disturbances like orthostatic hypotension and prolonged quadricep weakness secondary to peripheral nerve blocks. An attractive anesthetic option is a combined epidural-sedation technique. ${ }^{5}$ This multimodal approach avoids complete motor block, enabling patients to move their feet during the surgical procedure. When deep sedation ceases, patients can move their lower limbs while in bed, stand and walk 2-3 hours after surgery. This approach also prevents the increase of cortisol and adrenocorticotrophic hormone levels. Avoiding benzodiazepine likely improves patient awareness and responsiveness.

\section{Blood conservation}

There is a risk of substantial perioperative blood loss with THA and TKA surgeries. Preoperative hemoglobin optimization, perioperative systemic tranexamic acid administration, careful surgical hemostasis, keeping the TKA knee flexed for 3 hours postoperation and adrenalin in the local anesthetic infiltration, help to minimize blood loss and may also help to avoid the need for transfusion. ${ }^{5}$ Because followup of the postoperative hemoglobin level is not easily accessible from home, avoiding severe anemia is of primary importance when patients are discharged early after surgery.

\section{Prevention of thromboembolic events}

Because of surgical trauma and perioperative immobilization, THA and TKA surgeries activate the coagulation cascade and may increase the risk of blood clots or deep vein thrombosis. Perioperative intermittent compression of the lower limbs, early mobilization and oral anticoagulant therapy are essential elements of an ERAS program.

\section{Wound care}

Instead of using metallic staples, one option suggested by the ERAS program is to seal the wound with medical glue. This helps prevent wound discharge, spontaneous evacuation of hematomas, patient anxiety (i.e., misperception of hemorrhage) and soiling of the dressing. Skin closure with glue also reduces nursing care time and enables patients to shower and heal more quickly. ${ }^{5}$

\section{EVALUATION OF AN ERAS SHORT-STAY PROTOCOL}

In 2014-2015, Dr. Pascal-André Vendittoli and his team developed a perioperative short-stay protocol for THA and TKA procedures at Maisonneuve-Rosemont Hospital, following the ERAS principles. ${ }^{5}$ The key elements of the protocol were selected to be effective in reducing unwanted events (i.e., pain, adverse effects from opioids, patient sedation, anemia, wound complications, deep vein thrombosis, delayed function and mobilization and perioperative bowel malfunction). ERAS short-stay protocols require the involvement of a multidisciplinary team, including patients, anesthesiologists, surgeons, nurses, nutritionists and physiotherapists, who commit to specifically designed, evidence-based protocols in perioperative care. 
The team conducted a clinical study evaluating the rates of adverse events and the cost of this new clinical practice. ${ }^{5}$ Implementation of our ERAS short-stay protocol resulted not only in improved patient care, but also in reduced direct health care costs and duration of hospital stay. There was a significantly lower rate of grade 1 and 2 complications in the ERAS short-stay group compared with the standard group (mean 0.8 v. 3.0 complications, $p<0.001)$. The ERAS protocol was very efficient at reducing the frequency of patient complications, including pain, nausea, vomiting, dizziness, headache, constipation, hypotension, anemia, edema, lameness and urinary retention ( $p<0.001$ to $p=0.04$ ). The mean hospital length of stay for the ERAS short-stay group decreased by 2.8 days for THAs $(0.1$ v. $2.9 \mathrm{~d}, p<0.001)$ and 3.9 days for TKAs $(1.0$ v. $4.9 \mathrm{~d}, p<0.001)$. The mean estimated reduction in direct health care costs with the ERAS short-stay protocol was $\$ 1489$ per THA and $\$ 4158$ per TKA. Applied broadly and systematically, these savings would have a major impact for the Canadian health care system.

\section{Discussion}

To be effective, ERAS protocols should be applied systematically and include the patient and family at the core, supported through the efforts of the interdisciplinary team. In most cases, implementation involves important practice modifications. Following a common, clearly defined goal is key. Moreover, ERAS protocols should simplify postoperative care as much as possible. For example, the use of oral anticoagulants instead of injectables avoids the need for education on self-injection. Using skin glue to seal the wound reduces wound discharge and dressing changes, enables the patient to shower, eliminates the need for staple removal and results in less superficial infection. ${ }^{5}$

\section{Next steps for ERAS short-stay protocols in Canada}

Enhanced Recovery Canada (ERC) is an initiative of the Canadian Patient Safety Institute and was founded in 2017. Dr. Claude Laflamme, anesthesiologist at Sunnybrook Health Sciences Centre (Toronto, Ont.) is the current chair of the ERC Governance Committee. To date, ERC has focused on the development, dissemination and implementation of enhanced recovery-related best practices supporting elective colorectal surgeries. In recent months, ERC supported the development of ERC pathways for THA and TKA. Dr. Pascal-André Vendittoli was designated as the chair of the lead group, composed of other Canadian leaders in the field of outpatient TKA and THA. The goal of this group is to provide all Canadian health care providers and patients with helpful tools and resources to effectively implement and sustain ERAS protocols for TKA and THA procedures. ERC pathways for THA and
TKA will soon be available on the Canadian Patient Safety Institute website (www.patientsafetyinstitute.ca/en/ toolsResources/Enhanced-Recovery-after-Surgery/Pages/ default.aspx).

\section{Conclusion}

In various surgical specialties, following ERAS principles optimizes patient recovery, minimizes complications and improves the patient experience. ERAS should be the main goal for all patients, regardless of their surgical treatment. Applying these principles to a short-stay protocol for THA and TKA is the key to improved patient outcomes by reducing the rate of adverse events while reducing the duration of hospital stays to less than 24 hours. Shorter hospital stays after THA and TKA increases bed availability in a financially constrained environment. ERAS is also favourable for the care provider. It is a win-win situation for all.

Affiliations: From the Department of Surgery, University of Montréal, Montréal Que. (Venditolli); the Department of Surgery, MaisonneauveRosemont Hospital, Montréal Que. (Vendittoli, Pellei); Canadian Patient Safety Institute, St. John's, NL (Williams); and Sunnybrook Health Sciences Centre, Toronto, Ont. (Laflamme).

Competing interests: P.-A. Vendittoli reports grants and personal fees from Stryker and Johnson and Johnson, grants from Zimmer and from Smith and Nephew, and royalties from Microport, outside the submitted work. C. Williams is a senior program manager at the Canadian Patient Safety Institute and organizational lead for Enhanced Recovery Canada. No other competing interests are declared.

Contributors: All authors contributed substantially to the conception, writing and revision of this article and approved the final version for publication.

Content licence: This is an Open Access article distributed in accordance with the terms of the Creative Commons Attribution (CC BYNC-ND 4.0) licence, which permits use, distribution and reproduction in any medium, provided that the original publication is properly cited, the use is noncommercial (i.e., research or educational use), and no modifications or adaptations are made. See: https://creativecommons. org/licenses/by-nc-nd/4.0/

\section{References}

1. Kehlet H, Joshi GP. Enhanced recovery after surgery: current controversies and concerns. Anesth Analg 2017;125:2154-5.

2. Kehlet H, Slim K. The future of fast-track surgery. Br f Surg 2012;99:1025.

3. Wainwright TW, Gill M, McDonald DA et al. Consensus statement for perioperative care in total hip replacement and total knee replacement surgery: Enhanced Recovery After Surgery (ERAS) Society recommendations. Acta Orthop 2020;91:3-19.

4. Bodrogi A, Dervin GF, Beaulé PE. Management of patients undergoing same-day discharge primary total hip and knee arthroplasty. CMA7 2020;192:E34-9.

5. Vendittoli PA, Pellei K, Desmeules F et al. Enhanced recovery short-stay hip and knee joint replacement program improves patients outcomes while reducing hospital costs. Orthop Traumatol Surg Res 2019;105:1237-43. 